\title{
Does low daily energy expenditure drive low metabolic capacity in the tropical robin, Turdus grayi?
}

\author{
Dominique N. Wagner - Patrick M. Mineo • \\ Carrie Sgueo $\cdot$ Martin Wikelski $\cdot$ Paul J. Schaeffer
}

\begin{abstract}
Temperate and tropical birds possess divergent life history strategies. Physiological parameters including energy metabolism correlate with the life history such that tropical species with a slower 'pace of life' have lower resting and maximal metabolic rates than temperate congeners. To better understand the physiological mechanisms underlying these differences, we investigated the relationship of metabolic capacity, muscle oxidative capacity and activity patterns to variation in life history patterns in American robins (Turdus migratorius), while resident in central North America and Clay-colored robins (Turdus grayi) resident in Panama. We measured summit metabolism $\left(\dot{V} \mathrm{O}_{2 \text { summit }}\right)$ in birds from both tropical and temperate habitats and found that the temperate robins have a $60 \%$ higher metabolic capacity. We also measured the field metabolic rate (FMR) of free-living birds using heart rate (HR) telemetry and found that temperate robins' daily energy expenditure was also $60 \%$ higher. Thus, $\dot{V} \mathrm{O}_{2 \text { summit }}$ and FMR both reflect life history differences between the species. Further, both species operate at a nearly identical $\sim 50 \%$ of their thermogenic capacity throughout a given
\end{abstract}

day. As a potential mechanism to explain differences in activity and metabolic capacity, we ask whether oxidative properties of flight muscle are altered in accordance with life history variation and found minimal differences in oxidative capacity of skeletal muscle. These data demonstrate a close relationship between thermogenic capacity and daily activity in free-living birds. Further, they suggest that the slow pace of life in tropical birds may be related to the maintenance of low activity rather than functional capacity of the muscle tissue.

Keywords Avian life history - Muscle oxidative capacity . Phenotypic plasticity - Physiology-life history nexus .

Heart rate telemetry $\cdot$ Energetics

\section{Introduction}

Species differ widely in life history traits, reflecting differences in both the environment and in adaptive physiological responses (Stearns 1992; Robinson et al. 2010). We refer to the intersection of environmental factors and physiological mechanisms influencing demographic patterns of populations as the 'physiology-life history nexus' (Ricklefs and Wikelski 2002). There is a long history of observed differences in life history characteristics, especially in birds, over latitudinal gradients leading to the hypothesis that the relatively slower rate of reproduction typical of tropical environments selects for a slower pace of life (Wikelski and Ricklefs 2001; Cardillo 2002). This slower pace of life in tropical birds is characterized by a proportionally larger investment in self-maintenance rather than production of offspring, and manifests itself in longer life span and lower reproductive rate (Robinson et al. 2010). Further, physiological characteristics of tropical, 
compared to temperate, birds include greater investment in immune response (Lee et al. 2008), a more robust stress response (Hau et al. 2010), lower serum antioxidant activity (Cohen et al. 2008) and lower metabolic rates, both basal (Tieleman et al. 2006; Wiersma et al. 2007b) and maximal (Wiersma et al. 2007a).

Along the temperate-tropical life history gradient, the faster pace of life at higher latitudes is associated with higher metabolic capacity (Wiersma et al. 2007a). However, it is unclear whether increased metabolic rates directly predict activity level (i.e., 'pace of life'). For example, Ricklefs et al. (1996) found no connection between basal metabolic rate (BMR) and daily energy expenditure (DEE) in birds, as has been shown in mammals (Ricklefs et al. 1996). Supporting such an association, a recent study of a lowland tropical forest bird (using heart rate telemetry), found that the DEE as well as frequency of activity were low (Steiger et al. 2009). Further, field metabolic rate (measured with doubly labeled water) of tropical House Wrens was lower than that of the temperate zone individuals of the same species (Tieleman et al. 2006). Desert larks have lower BMR than mesic congeners, suggesting a broad potential for adaptation of avian metabolism in response to environmental challenges (Williams and Tieleman 2005).

As there are no data for both metabolic capacity and DEE for tropical birds, the relationship between metabolic capacity and DEE remains unknown. However, sustainable metabolic rates establish an animal's metabolic ceiling (Piersma 2011), potentially limiting activity. With change in DEE, birds must change the fraction of available metabolic capacity used for activity, change capacity, or use a combination of these two strategies (Liknes et al. 2002). The explicit purpose of our study was to assess whether a fast pace of life is associated with elevation of this ceiling reflecting greater intensity of daily energetic output.

Organismal metabolic rate is a function of tissue properties; therefore, we expect that species with 'fast' life history will exhibit relatively higher skeletal muscle metabolic capacity in concordance with increased activity, as suggested by countless exercise training studies in mammals (reviewed by Fluck and Hoppeler 2003). In temperate resident birds, winter cold has been shown to increase tissue metabolic capacity (Swanson 1991; O’Connor 1995; Zheng et al. 2008) and increased flight muscle mass has also been documented in several migrant bird species (Piersma 1998; Lindström et al. 2000; Bauchinger et al. 2005), suggesting that activity level may be important in establishing differences in tissue metabolic capacity. While, life history driven variation in organismal pace of life may be related to variation in muscle oxidative capacity, we are unaware of any data addressing this question.
To investigate the relationship between metabolic capacity, muscle oxidative capacity and activity patterns as related to variation in life history patterns, we studied American robins (Turdus migratorius), while resident in central North America and Clay-colored robins (Turdus grayi) in Panama, hereafter abbreviated AMRO and CCTH, respectively, following standard ornithology alphacode abbreviations. Both species inhabit similar niches in their respective environments and possess differing life history strategies. For example, annual survival of the tropical CCTH is estimated at $\sim 77 \%$, while that of temperate AMRO is estimated at about $50 \%$ (Ricklefs 1997). Similarly, at about 3.5 eggs per nest, clutch size is higher in AMRO (Ricklefs and Bloom 1977; Henny 1972) than CCTH, which lay about 2.7 eggs per nest (Skutch 1981). Temperate AMRO are more successful at fledging young, with double the number of individual eggs that lead to surviving fledglings and a doubling in overall seasonal reproduction (Ricklefs 1997), despite that the tropical breeding season is longer (Stutchbury et al. 1998). We first asked whether this species pair exhibits the previously described temperate-tropical pattern in metabolic capacity reflecting differences in life history by measuring summit metabolic rate $\left(\dot{V} \mathrm{O}_{2 \text { summit }}\right)$ achieved by cold heliox $\left(\mathrm{HeO}_{2}\right)$ exposure as described by Rosenmann and Morrison (1974). We next ask whether DEE reflects maximum metabolic capacity and life history predictions by measuring field metabolic rates of free-living birds in both tropical and temperate habitats using heart rate (HR) telemetry. HR telemetry permits measurement of DEE with accuracy similar to the doubly labeled water approach (Butler et al. 2004), as well as finer scale description of metabolic rate during specific behaviors or activity periods (Bisson et al. 2009; Sgueo et al. 2012). Given the limited data on field metabolic rates of tropical birds, how metabolic capacity is related to daily activity rates remains unknown. Finally, as a potential mechanism to explain differences in activity and metabolic capacity, we asked whether oxidative properties of flight muscle are altered in accordance with life history variation by comparing histological measurements of muscle fiber type (myosin ATPase) and succinate dehydrogenase activity, and biochemical measurement of citrate synthase activity.

\section{Materials and methods}

\section{Study species and field sites}

We studied American robins (hereafter-AMRO), a migratory species, abundant in Michigan and Ohio (the north-central U.S.) and Clay-colored robins (hereafter- 
CCTH), resident in the Neotropics, including Panama. These congeners prefer open, disturbed habitat, generally grassy fields, and feed on invertebrates found on or near the surface. Foraging activity is almost, exclusively, limited to dawn and dusk in both species with individuals shading in trees during midday. While both species have similar dietary habits, it is unknown exactly what species are targeted or whether foraging efforts vary. Study sites were located at the Kellogg Biological Station in south-central Michigan $\left(\mathrm{KBS} ; 42^{\circ} 17^{\prime} \mathrm{N}, 85^{\circ} 36^{\prime} \mathrm{W}\right)$, at The Ecology Research Center of Miami University (ERC; $39^{\circ} 30^{\prime} \mathrm{N}$ and $84^{\circ} 45^{\prime} \mathrm{W}$ ) in southwest Ohio and at the Smithsonian Tropical Research Institute in Gamboa, Panama (STRI; $9^{\circ} \mathrm{N}, 79^{\circ} \mathrm{W}$ ). Birds were caught with mist nets between sunrise and late afternoon, and released at least $1 \mathrm{~h}$ before sunset to facilitate a successful transition back to the environment following laboratory interventions.

For measurement of summit metabolism, AMRO were captured in July 2005 at KBS and CCTH at STRI in March 2006. In all cases, sampling was done during the breeding season for these species; however, we did not verify breeding status for individuals used in this study. Birds were captured under either permission from Kellogg Biological Station, Michigan, (to RE Ricklefs), US Fish and Wildlife Service banding permit (to MW), and permission from the Autoridad del Ambiente Nacional (ANAM, Republic of Panama) and the Smithsonian Tropical Research Institute (STRI; to MW). Upon capture, birds were either first bled $(\sim 200 \mu \mathrm{l})$ for other studies or immediately taken to laboratory facilities and housed. Birds that have been previously bled were maintained for $24 \mathrm{~h}$ before other measurements. Birds were housed individually in cages with ad lib access to mealworms, berries and water and maintained with approximately normal seasonal light:dark cycles.

For HR telemetry or tissue analysis, AMRO were captured at the ERC in July-August 2007 (average high temperature of $31{ }^{\circ} \mathrm{C}$, average low temperature of $18{ }^{\circ} \mathrm{C}$ ) and May-June 2008 (average high temperature of $30{ }^{\circ} \mathrm{C}$, average low temperature of $17^{\circ} \mathrm{C}$ ) with permission from the Ohio Department of Natural Resources, US Fish and Wildlife Service (to PJS) and, CCTH at STRI in June 2007 and July 2008 (average high temperatures of 30 and $31^{\circ} \mathrm{C}$ and average low temperatures of 23 and $24{ }^{\circ} \mathrm{C}$, respectively) with permission from the ANAM and STRI (to PJS). These animals were taken directly to the laboratory and experiments performed immediately. All animal experimentation was approved by the Institutional Animal Care and Use Committee of Miami University (protocol \#722) as well as Princeton University (protocols 1428, 1429 and 1516) and complied with the "Principles of Animal Care," publication no. 86-23, revised 1985, of the National Institutes of Health as well as the laws of the
United States. All the capture and handling of Panamanian animals were done in concordance with Panamanian laws and under permits from Panamanian authorities.

\section{Physiology experiments}

\section{Field metabolic rate}

Once birds were brought into the laboratory, a HR radiotransmitter was attached to the mid-scapular region of the back of the bird with fine wire electrodes placed subcutaneously for detection and transmission of ECG signal as described previously (Cochran and Wikelski 2005; Froget et al. 2001; Steiger et al. 2009; Sgueo et al. 2012). Transmitters ( $\sim 1$ gram; J.D.J.C. Corp., Fisher, IL, USA) weighed less than $2 \%$ of the bird's body mass, minimizing the likelihood that birds were experiencing increased metabolic effort in carrying transmitters (Gow et al. 2011, but see Barron et al. 2010). After transmitter attachment, and before bird release, we simultaneously measured HR and oxygen consumption using indirect calorimetry (Sable Systems, Las Vegas, NV, USA) to generate a calibration curve (Bisson et al. 2009; Sgueo et al. 2012). During each measurement, the bird was placed into an approximately 4.5 liter plastic chamber with constant flow of room air. Positive pressure gas flow (room air) was regulated by a mass flow meter (0-5 L/ min range; Sierra Instruments, Inc., Monterey, CA) controlled by a mass flow controller (model MFC-2; Sable Systems, Las Vegas, NV) at $1.4 \mathrm{~L} / \mathrm{min}$. Gas passed through the chamber containing the bird $(4.5 \mathrm{~L}$ volume for FMR calibration or $1.2 \mathrm{~L}$ for $\dot{V} \mathrm{O}_{2 \text { summit }}$, below) and then passed through a $\mathrm{CO}_{2}$ analyzer (model CA-10a; Sable Systems, Las Vegas, NV), a drierite (dessicant) column and an $\mathrm{O}_{2}$ analyzer (model FC-10a; Sable Systems, Las Vegas, NV). Calibration of both $\mathrm{O}_{2}$ and $\mathrm{CO}_{2}$ analyzers followed the instructions in the Sable Systems manuals using ambient air and $100 \%$ nitrogen as a 'zero'. Washout times were 9 and $4 \mathrm{~min}$, respectively, for FMR or $\dot{V} \mathrm{O}_{2 \text { summit }}$ (below). In all cases, birds were held at room temperature during measurements, which lasted from 40 to $60 \mathrm{~min}$ per animal. To reduce the equilibration time, birds were placed into chambers at room temperature and gases equilibrated at rest before beginning experiments. To generate a wide range of both HRs and oxygen consumption rates that reflect physiological limits, measurements were made while the bird was at rest as well as while the bird was agitated (by drumming on, moving and shaking the chamber), either mildly or severely, in the metabolic chamber. While an elevation in $\mathrm{HR}$ and oxygen consumption in response to stress is not equivalent to elevation due to "natural" activity, the 
birds became very active under these stimuli, moving and fluttering wings and generated a wide range of heart rate and metabolic rate values used for calibration of HR to oxygen consumption. These equations typically had an $\mathrm{r}^{2}$ of about 0.9. Each phase of calibration extended over at least $10 \mathrm{~min}$, once steady state for oxygen and carbon dioxide readouts were reached and several HR measurements were taken. The range of HRs obtained during calibration spanned HRs obtained in the field. Oxygen consumption rates $\left(\dot{V} \mathrm{O}_{2}\right)$ at each activity level were calculated from $1 \mathrm{~min}$ of steady state data using the following equation from the Sable Systems manual:

$$
\begin{aligned}
\dot{V} \mathrm{O}_{2}= & \mathrm{STP} \cdot \mathrm{FR} \\
& \cdot\left(\left(\mathrm{F}_{\mathrm{i}} \mathrm{O}_{2}-\mathrm{F}_{\mathrm{e}} \mathrm{O}_{2}\right)-\mathrm{F}_{\mathrm{e}} \mathrm{O}_{2} \cdot\left(\mathrm{F}_{\mathrm{e}} \mathrm{CO}_{2}-\mathrm{F}_{\mathrm{i}} \mathrm{CO}_{2}\right)\right) \\
& /\left(1-\mathrm{F}_{\mathrm{e}} \mathrm{O}_{2}\right)
\end{aligned}
$$

where $\mathrm{STP}=$ standard temperature and pressure; $\mathrm{FR}=$ flow rate $; \mathrm{F}_{\mathrm{i}} \mathrm{O}_{2}$ and $\mathrm{F}_{\mathrm{i}} \mathrm{CO}_{2}=$ fractional content of oxygen and carbon dioxide, respectively, in incurrent air; $\mathrm{F}_{\mathrm{e}} \mathrm{O}_{2}$ and $\mathrm{F}_{\mathrm{e}} \mathrm{CO}_{2}=$ fractional content of oxygen and carbon dioxide, respectively, in excurrent air. Individual calibration curves were collected for each bird and used to determine field metabolic rate from HR data collected in the field as described below. For two AMRO, we were unable to generate an individual calibration curve and used a master calibration curve produced by combining data from all individual AMRO (including some that we were unable to track). Use of master calibration curves for all animals produced nearly identical values for DEE and differences between groups that were also statistically significant.

Following collection of the calibration data, birds were released at the site of capture. Radio-telemetry was used to track each individual for 24 continuous hours, beginning at least $12 \mathrm{~h}$ after release, enabling the bird to recover from transmitter attachment and achieve a standard physiological state. During the 24-h tracking period, HR was recorded every $5 \mathrm{~min}$ and was assumed to be representative for that period. We successfully collected telemetric ECG for a complete day from four AMRO and six CCTH. HR was determined following transformation (FFT) and filtering of raw transmissions using CoolEdit 2000, as described previously (Bisson et al. 2009). Field metabolic rate calculated as rate of oxygen consumption and expressed as DEE, was then determined using the mean HR from field measurements and the laboratory calibration equation. In addition to DEE, rate of energy use was calculated on an hourly basis to observe the pattern of oxygen consumption throughout a given day.

\section{Summit metabolism}

A second set of birds was used to measure thermogenic capacity $\left(\dot{V} \mathrm{O}_{2 \text { summit }}\right)$ as a surrogate for maximal metabolic capacity. After weighing, summit metabolism measurements (following Rosenmann and Morrison 1974).were collected, while birds breathed a helium-oxygen gas mixture $($ Flow $=1.2 \mathrm{~L} / \mathrm{min}$; chamber volume $\sim 1 \mathrm{~L}$ ). The heliox $\left(\mathrm{HeO}_{2}\right)$ environment facilitates rapid body heat loss while minimizing the danger of freezing damage to tissues. Birds were initially left at room temperature for 10-15 min to allow system equilibration before being placed into a static $4{ }^{\circ} \mathrm{C}$ temperature (Swanson et al. 1996). The $\mathrm{CO}_{2}$ content of excurrent air was closely monitored and the bird was removed upon decline of $\mathrm{CO}_{2}$ content. Immediately upon completion of the session, cloacal temperature at a standard depth that had been previously determined to accurately measure core temperature. In all cases, birds were slightly hypothermic $\left(\mathrm{T}_{\mathrm{b}} \leq 38{ }^{\circ} \mathrm{C}\right)$. $\dot{V} \mathrm{O}_{2 \text { summit }}$ was calculated using the peak value calculated from $1 \mathrm{~min}$ of respiration data once the bird had reached a steady state plateau using Eq. (1).

\section{Metabolic 'effort'}

We divided values for field metabolic rate by summit metabolism (expressed as DEE/Summit) to determine the relative metabolic effort, or the extent to which birds utilized metabolic capacity, noting that this value is likely an overestimate as our measure of metabolic capacity is somewhat below maximum achievable during flight in a wind tunnel.

\section{Tissue experiments}

A third set of birds was anesthetized with inhaled isofluorane and decapitated for tissue collection. Samples were dissected from the medial region of the pectoralis muscle from each individual, frozen in 2-methyl butane in liquid nitrogen for histology or directly frozen in liquid nitrogen for biochemical assay and stored at $-80{ }^{\circ} \mathrm{C}$ for later analysis.

\section{Muscle fiber type histology}

Frozen pectoralis muscle samples were mounted in tragacanth gum, sectioned (12 microns) in a Microm HM505 N cryostat (Carl Zeiss, Waldorf, Germany) and placed on glass slides. Staining for myosin ATPase activity followed Brooke and Kaiser (1970). Pre-incubation pH was 4.53, 
4.60 and 10.60 to visualize type IIa, IIb and I, respectively. We were unable to differentiate between type IIa and IIb; however, using a range of similar acid pre-incubation steps, thus, data are reported using the 4.53 pre-incubation step in which only type I fibers stain darkly. Succinate dehydrogenase staining followed Sheehan and Hrapchak (1987). Briefly, sections were prepared as above and incubated in phosphate buffer with succinic acid and a redox indicator (nitro blue tetrazolium). Mitochondrial oxidative activity results in dark blue fibers. In both cases, stained and mounted sections were systematically photographed with a stereomicroscope (Olympus SZX-12; Olympus Corp., Center Valley PA, USA) at $100 \times$ magnification. Total number of complete cells within each field was counted for each stain and percentage appearance of each cell type calculated. For SDH staining, we analyzed sections from five individuals of each species.

\section{Biochemical assays}

Our analysis of muscle oxidative capacity used a citrate synthase assay modified from Chi et al. (1983). Samples were prepared by hand homogenization (on ice) with 19 volumes of homogenization medium (0.05 M Tris, 0.15 M $\mathrm{KCL}, \mathrm{pH}$ 7.4) followed by centrifugation. Supernatant was removed, frozen in liquid nitrogen and stored at $-80{ }^{\circ} \mathrm{C}$. To assay maximal citrate synthase activity, pectoralis homogenates were added to assay reagent $(0.1 \mathrm{M}$ Tris, pH8.1) with an excess of Acetyl-CoA (0.02 mM) and Oxaloacetate $(0.17 \mathrm{mM})$. The rate of disappearance of Acetyl-CoA was monitored at $232 \mathrm{~nm}$ in a temperaturecontrolled spectrophotometer (DTX880 Multimode Detector, Beckman Coulter Inc., Fullerton, CA) at $25{ }^{\circ} \mathrm{C}$ to determine the rate of enzyme activity. Pectoralis samples were analyzed for seven AMRO and six CCTH.

\section{Statistical analysis}

We used students's $t$ tests for pairwise comparisons of physiological variables between AMRO and CCTH for summit metabolism, all histology measurements, muscle oxidative capacity and DEE. Hour-by-hour energy use rates were analyzed using repeated measures ANOVA in SAS (version 9.1 for Windows). The level of significance was set at $p<0.05$ in all cases, except for pairwise comparisons of individual $1 \mathrm{~h}$ periods of rate of energy use for which we used a Bonferroni correction. As data for DEE and summit metabolism were not collected on the same individuals, ratios of DEE/Summit were analyzed using bootstrapping (10,000 iterations) to generate a nonparametric $95 \%$ confidence interval for the true mean ratio. The analysis was performed using $\mathrm{R}$ statistical software (version 2.9.0). Data are presented as mean \pm SEM [n].

\section{Results}

Body mass

AMRO body mass (70.5 $\pm 1.6 \mathrm{~g}$ [8]) was significantly greater than $\mathrm{CCTH}$ body mass $(58.5 \pm 1.9 \mathrm{~g}$ [8]; $p<0.001)$. Given this difference, we report measures of metabolic rate as both whole animal and mass-specific measures.

\section{Thermogenic capacity}

Whole animal summit metabolism was significantly greater $(88.9 \%)$ in AMRO than CCTH $(p<0.001$; Table 1$)$. Although the AMRO had a higher body mass than the $\mathrm{CCTH}$, and thus should have lower mass-specific metabolism according to the body mass scaling of metabolic rates, their mass-specific summit metabolism was also significantly greater $(60.0 \%)$ than CCTH $(p<0.01$; Table 1).

\section{Muscle histology and biochemistry}

Myosin ATPase staining showed no significant difference in the proportion of fast vs. slow twitch fibers (Fig. 1). Flight muscle of both species was composed of approximately $36 \%$ slow, type I, fibers (AMRO, $35.8 \pm 2.0$ [7] \%; CCTH, $36.2 \pm 2.4$ [6] \%) and $64 \%$ fast fibers (AMRO, $64.2 \pm 2.0$ [7] \%; CCTH, $63.8 \pm 2.4$ [6] \%). We were unable to differentiate between types of fast fiber in this assay, thus the proportion reported as fast fibers are a combination of both type IIa and IIb.

There was a significant increase in the percentage of those fibers staining for succinate dehydrogenase (SDH) activity, a marker of mitochondrial function, i.e., oxidative fibers, in the pectoralis muscle of the temperate robins (AMRO, $70.9 \pm 1.7$ [5] \% vs. CCTH, $61.0 \pm 2.6$ [5] \%; $p<0.05$ ), suggesting a more oxidative phenotype (Fig. 2).

To more directly measure mitochondrial capacity of pectoralis muscle, we measured citrate synthase activity as a marker of the tissue mitochondrial capacity. Although similar to the SDH results, citrate synthase activity was not significantly different between species. Therefore, it appears that the metabolic capacity of the pectoralis muscle is similar in the two species (Table 1).

\section{Field metabolic rate}

To determine whether the "pace of life" is reflected in the daily energy budget, we measured field metabolic rate using HR telemetry of free-living animals. We used these data to determine the DEE of AMRO in Ohio and CCTH in Panama. DEE was significantly higher in the AMRO 
Table 1 Metabolic measurements of temperate (T. migratorius-AMRO) and tropical (T. grayi-CCTH) robins

\begin{tabular}{|c|c|c|}
\hline & AMRO & ССТH \\
\hline $\operatorname{DEE}\left(\mathrm{LO}_{2} \mathrm{day}^{-1}\right)$ & $7.80 \pm 0.55[4]$ & $4.77 \pm 0.33[6]^{*}$ \\
\hline Mass-specific DEE $\left(\mathrm{LO}_{2} \mathrm{~kg}^{-1}\right.$ day $\left.^{-1}\right)$ & $104.6 \pm 6.7[4]$ & $65.8 \pm 4.3[6]^{*}$ \\
\hline$\dot{V} \mathrm{O}_{2 \text { summit }}\left(\mathrm{mlO}_{2} \min ^{-1}\right)$ & $10.30 \pm 0.88[8]$ & $5.40 \pm 0.63[8]^{*}$ \\
\hline Mass-specific $\dot{V} \mathrm{O}_{2 \text { summit }}\left(\mathrm{mlO}_{2} \mathrm{~kg}^{-1} \min ^{-1}\right)$ & $145.2 \pm 11.9[8]$ & $90.8 \pm 8.0[8]^{*}$ \\
\hline $\mathrm{DEE} / \dot{V} \mathrm{O}_{2 \text { summit }}(\%)$ & $52.5,2.6$ & $53.4,2.3$ \\
\hline Citrate synthase activity $\left(\mu \mathrm{mol} \mathrm{g}{ }^{-1} \min ^{-1}\right)$ & $112.2 \pm 9.4[7]$ & $102.7 \pm 5.9[6]$ \\
\hline
\end{tabular}

Following predictions from life history theory, both measures of organismal metabolic rate, daily energy expenditure (DEE) and summit metabolism $\left(\dot{V} \mathrm{O}_{2 \text { summit }}\right)$, are higher in temperate robins as compared to tropical robins. However, both species are operating at the same percentage of their capacity (DEE/V $\mathrm{O}_{2 \text { summit }}$, calculated using whole animal values). We observed no difference in pectoralis muscle citrate synthase activity. DEE was determined in the field via heart rate telemetry. $\dot{V} \mathrm{O}_{2 \text { summit }}$ was determined via indirect calorimetry in a heliumoxygen atmosphere. Data are presented as mean $\pm \mathrm{SEM}[\mathrm{n}]$, except for the ratio of DEE/V $\mathrm{O}_{2 \text { summit }}$ which is presented as mean, bootstrap standard error

*indicates significant difference from temperate robins at $p<0.05$ level
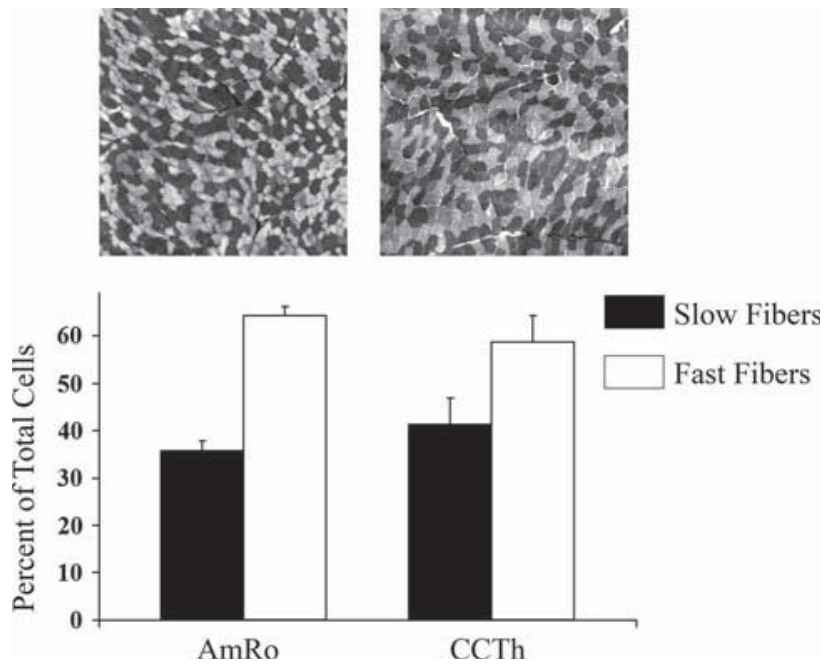

Fig. 1 Flight muscle fiber type composition is similar in American and Clay-colored robins. Histology revealed no significant difference in muscle fiber types using ATPase staining. Flight muscle of both species was approximately $35 \%$ slow, type I and $65 \%$ fast fibers. Values are mean \pm SEM

whether expressed per whole animal $(64.0 \%, p<0.01$; Table 1) or as a mass-specific measure $(59.0 \%, p<0.01$; Table 1). These results indicate that, like metabolic capacity, the "fast" species uses about $60 \%$ more energy per day than their "slow" relatives do.

One strength of the HR approach for determination of DEE is the ability to examine hour-by-hour patterns in energy use. We calculated hourly rates of oxygen consumption for AMRO and CCTH for a 24-h period (Fig. 3). ANOVA revealed that there was no significant interaction between robin species and time, indicating that the effect of robin species on DEE does not significantly change by time $(F[23,237]=1.04, p=0.4169)$. There was a significant effect of robin species regardless of time $(F[1,13]=31.14$,
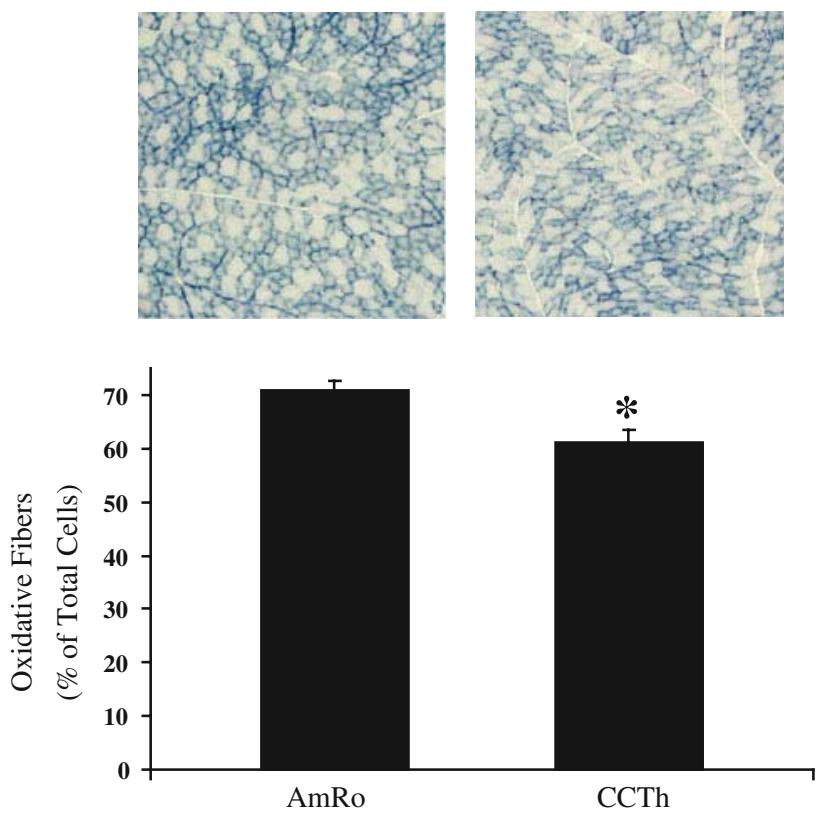

Fig. 2 The percentage of oxidative fibers is higher in the American robin than the Clay-colored robin. The percentage of pectoralis muscle fibers staining for succinate dehydrogenase activity was significantly higher in the American robin $(p<0.05)$. Values are mean \pm SEM and asterisks indicate significant differences between groups

$p<0.0001$ ), such that the DEE of AMRO was greater than the CCTH. This field metabolic rate was greater for AMRO during the daylight hours, and most of the nighttime hours with the exception of the 4th, 5th, 6th and 17th hours of the day. Therefore, the higher hourly rates of energy expenditure observed in AMRO are not limited to the time periods that the birds are most active, but are also observed during much of the inactive portion as well.

Lastly, we compared the ratio of daily energy expenditure and metabolic capacity (DEE/Summit) between 


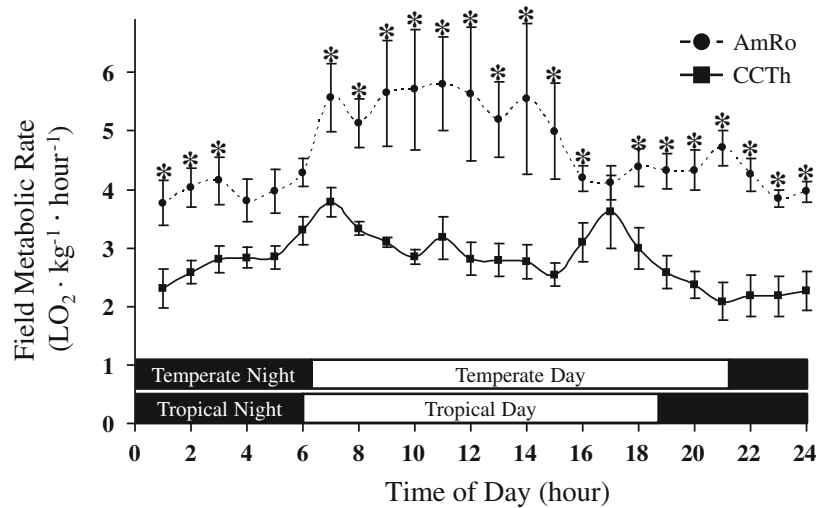

Fig. 3 Energy expenditure is higher in American robins throughout the day. American robins had higher metabolic rates than the Claycolored robins at all periods except hours 4,5 and 6 (all-overnight hours) and hour 17. Thus, the higher metabolic rate of American robins extends throughout the entire day and through much of the night as well. Values are mean \pm SEM and asterisks indicate significant differences between groups at each time point

species. Average daily energy use rate is undertaken as a percentage of metabolic capacity and, thus, represents the relative effort of each species during free-living behavior in their native habitat. There was no significant difference in the ratio of DEE/Summit between species (bootstrap ratio of 1.02 with a $95 \% \mathrm{CI}=0.91-1.14$; Table 1), indicating that DEE is a similar percentage of metabolic capacity in both species.

\section{Discussion}

We found that tropical robins had lower metabolic capacity as measured by summit metabolism, consistent with a previous report examining several lowland tropical rainforest species (Wiersma et al. 2007a) and previous predictions (e.g., Vleck and Vleck 1979; Hails 1983; McNab 2001). Following our expectation that higher capacity would be linked to higher activity and daily rates of energy use, field metabolic rate measurements using HR telemetry showed higher DEE in the temperate robins, similar to that seen in temperate wrens using doubly labeled water (Tieleman et al. 2006). As AMRO are significantly larger than CCTH, body mass allometry would predict a higher mass-specific metabolic rate in the smaller tropical CCTH. However, CCTH had lower whole animal and mass-specific metabolic rates, in agreement with the proposal that life history drives the differences in the energetic pace of life of these two species. The lower nighttime temperatures in Ohio may have also contributed to the observed differences. While we present a two species comparison, these species are closely related congeners and ecologically similar, providing a reasonable system to test whether differences in their life history (Ricklefs 1997; Stutchbury et al. 1998) can drive physiological adaptation of their energy metabolic systems.

Measurement of both thermogenic capacity $\left(\dot{V} \mathrm{O}_{2 \text { summit }}\right)$ and DEE in the same study permits calculation of the relative efforts of each species (as the ratio of DEE/Summit). Note that summit metabolic rate is lower than maximal metabolic rate measured while flying (McKechnie and Swanson 2010); however, this approach is a tractable measure of metabolic capacity as it requires no training and animals become hypothermic, identifying a clear endpoint. In mammalian studies, $\dot{V} \mathrm{O}_{2 \text { summit }}$ correlates with running $\dot{V} \mathrm{O}_{2 \max }$ (e.g., Conley et al. 1985, Schaeffer et al. 2003), such that $\dot{V} \mathrm{O}_{2 \text { summit }}$ is $70-80 \%$ of $\dot{V} \mathrm{O}_{2 \max }$. In the one study to date, that examined both $\dot{V} \mathrm{O}_{2 \text { summit }}$ and $\dot{V} \mathrm{O}_{2 \max }$ in birds, this relationship was not observed (Wiersma et al. $2007 \mathrm{a}$, b). However, $\dot{V} \mathrm{O}_{2 \max }$ was measured using the 'hop-flutter wheel' in which exhaustion of the birds, rather than a physiological endpoint such as lactate accumulation was the identified endpoint. Thus, variable motivation may have confounded the conclusion. Using this approach, we found that both species operate at a nearly identical $\sim 50 \%$ of their thermogenic capacity throughout a given day. The close matching of these two variables supports the hypothesis that life history establishes both daily activity levels and metabolic ceilings (Piersma 2011) that permit that activity. For any organism to increase its DEE, it must either increase the percent of its capacity that is used or increase capacity (Liknes et al. 2002). It is striking that each of these species has such a similar relationship between use and capacity, which argues that metabolic capacity and DEE are closely coupled in birds, unlike BMR and DEE (Ricklefs et al. 1996). Both species operate at slightly higher percentage of capacity than Northern cardinals (Cardinalis cardinalis; Sgueo et al. 2012). However, we acknowledge that phenotypic flexibility (Piersma and Lindström 1997) may contribute as differences in activity may drive variation in physiological capacity through acclimatization without adaptive, evolutionary change of metabolic capacity. These measures of metabolic capacity and DEE do not permit us to determine whether CCTH could respond to greater demand by increasing metabolic capacity as has been demonstrated in many mammalian training studies (reviewed by Fluck and Hoppeler 2003). Nonetheless, a slower pace of life has resulted in lower DEE and summit metabolism in CCTH such that they operate at a percentage of capacity that is identical to that seen in the temperate AMRO.

Our measurement of field metabolic rate using HR telemetry permits fine scale determination of hourly patterns of daily energy use (Bisson et al. 2009; Steiger et al. 2009; Bowlin and Wikelski 2008; Sgueo et al. 2012). 
While AMRO had higher hourly energy expenditure throughout the day, the differences were particularly pronounced during the daylight hours. The higher error measures of the AMRO during the daylight hours reflect the fact that some, but not all the birds were active at any given period, while CCTH largely restricted activity to crepuscular periods. Our data reveal a profound difference in patterns of activity and energy use between the temperate and tropical member of the genus.

Differences in both the whole animal energy use and capacity are expected to be a feature of tissue differences, as already suggested (Kleiber 1961). We examined histological and biochemical markers of oxidative capacity in the flight muscle of both species, but found little evidence that this muscle is responsible for the organismal differences observed. Fiber type staining for myosin isoform (an indirect measure of ATP use rate) showed no difference. Histological and biochemical measures of mitochondrial function were equivocal, with only the SDH histological staining reaching significance. However, while both tending to be higher in the temperate AMRO, the difference was much smaller than whole organismal differences. While several studies have shown that skeletal muscle oxidative capacity is increased with seasonal cold acclimatization (Swanson 1991; O'Connor 1995; Zheng et al. 2008) or migration (Piersma 1998; Lindström et al. 2000; Bauchinger et al. 2005), others have failed to do so (Vézina et al. 2006; Sgueo et al. 2012), thus, the contribution of muscle plasticity to seasonal acclimatization remains unresolved.

The demands of divergent life history have apparently led to the evolution of several physiological systems (Robinson et al. 2010). We present evidence supporting the hypothesis that a faster pace of life is associated with a coordinated increase in DEE and summit metabolism, and our data highlight the mechanisms by which such differences are achieved. Temperate robins operate at higher metabolic rates throughout the day and possess higher metabolic capacity to support this activity. While evolution can define energy metabolic physiology, the importance of phenotypic flexibility is also well documented (Piersma, 2011). We suggest that the tight coordination of daily use and capacity is indicative of a finely tuned and likely plastic system. However, whether divergent energy metabolism of temperate and tropical birds is the best described by adaptive or acclimative processes await common garden experiments.

Acknowledgments This work was funded by National Science Foundation grant (IRCEB IBN-0212587 The Life History-Physiology Nexus: Constraints on the Evolutionary Diversification of Avian Life Histories) to MW, a grant from the Hampton Fund and other funds from Miami University to PJS and an undergraduate research award to DNW. We thank Rodney Kolb and the staff at the ERC, KBS and
STRI field stations and our field crew including Brett Aiello, Brian Lin, Lauren Paluch and Patrick Walsh, without whose help this work could not have been done. We also thank Michael Hughes and Dr. A. John Bailer from the Miami University Statistical Consulting Center for assistance with statistical analysis.

\section{References}

Barron DG, Brawn JA, Weatherhead PJ (2010) Meta-analysis of transmitter effects on avian behavior and ecology. Methods Ecol Evol 1:180-187

Bauchinger U, Wohlmann A, Biebach H (2005) Flexible remodeling of organ size during spring migration of the garden warbler (Sylvia borin). Zoology 108:97-106

Bisson IA, Butler LK, Hayden TJ, Romero LM, Wikelski MC (2009) No energetic cost of anthropogenic disturbance in a songbird. Proc Biol Sci 276:961-969

Bowlin MS, Wikelski M (2008) Pointed wings, low wingloading and calm air reduce migratory flight costs in songbirds. PLoS ONE 3:e2154. doi:10.1371/journal.pone.0002154

Brooke MH, Kaiser KK (1970) Three myosin adenosine triphosphatase systems - nature of their $\mathrm{pH}$ lability and sulfhydryl dependence. J Histochem Cytochem 18:670-672

Butler PJ, Green JA, Boyd IL, Speakman JR (2004) Measuring metabolic rate in the field: the pros and cons of the doubly labeled water and heart rate methods. Funct Ecol 18:168-183

Cardillo M (2002) The life-history basis of latitudinal diversity gradients: how do species traits vary from the poles to the equator. J Anim Ecol 71:79-87

Chi MM, Hintz CS, Coyle EF, Martin WH, Ivy JL, Nemeth PM, Holloszy JO, Lowry OH (1983) Effects of detraining on enzymes of energy-metabolism in individual human-muscle fibers. Am J Physiol 244:C276-C287

Cochran WW, Wikelski M (2005) Individual migratory tactics of New World Catharus thrushes: current knowledge and future tracking options from space. In: Marra P, Greenberg R (eds) Birds of Two Worlds: The Ecology and Evolution of Migration. Johns Hopkins University Press, Baltimore, pp 274-289

Cohen AA, McGraw KJ, Wiersma P, Williams JB, Robinson WD, Robinson TR, Brawn JD, Ricklefs RE (2008) Interspecific associations between circulating antioxidant levels and lifehistory variation in birds. Am Nat 172:178-193

Conley KE, Weibel ER, Taylor CR, Hoppeler H (1985) Aerobic activity estimated by exercise vs cold-exposure: endurance training effects in rats. Respir Physiol 62:273-280

Fluck M, Hoppeler H (2003) Molecular basis of skeletal muscle plasticity-from gene to form and function. Rev Physiol Biochem Pharmacol 146:159-216

Froget G, Butler PJ, Handrich Y, Woakes AJ (2001) Heart rate as an indicator of oxygenconsumption: influence of body condition in the king penguin. J Exp Biol 204:2133-2144

Gow EA, Done TW, Stutchbury BJM (2011) Radio-tags have no behavioral or physiological effects on a migratory songbird during breeding and molt. J Field Ornithol 82:193-201

Hails C (1983) The metabolic rate of tropical birds. Condor 85:61-65

Hau M, Ricklefs RE, Wikelski M, Lee KA, Brawn JD (2010) Corticosterone, testosterone and life-history strategies of birds. Proc R Soc B 277:3203-3212

Henny CJ (1972) An analysis of the population dynamics of selected avian species. Wildl Res Rep 1:1-99

Kleiber M (1961) The fire of life: an introduction to animal energetics. John Wiley \& Sons, New York

Lee KA, Wikelski M, Robinson WD, Robinson TR, Klasing KC (2008) Constitutive immune defenses correlate with life-history variables in tropical birds. J Anim Ecol 77:356-363 
Liknes ET, Scott SM, Swanson DL (2002) Seasonal acclimatization in the American goldfinch revisited: to what extent do metabolic rates vary seasonally? Condor 104:548-557

Lindström A, Kvist A, Piersma T, Dekinga A, Dietz MW (2000) Avian pectoral muscle size rapidly tracks body mass changes during flight, fasting and fueling. J Exp Biol 203:913-919

McKechnie AE, Swanson DL (2010) Sources and significance of variation in basal, summit and maximal metabolic rates in birds. Curr Zool 56:741-758

McNab BK (2001) Energetics of toucans, a barbet, and a hornbill: implications for avian frugivory. Auk 118:916-933

O'Connor TP (1995) Metabolic characteristics and body composition in house finches: effects of seasonal acclimatization. J Comp Physiol B 165:298-305

Piersma T (1998) Phenotypic flexibility during migration: optimization of organ size contingent on the risks and rewards of fueling and flight? J Avian Biol 29:511-520

Piersma T (2011) Why marathon migrants get away with high metabolic ceilings: toward an ecology of physiological restraint. J Exp Biol 214:295-302

Piersma T, Lindström A (1997) Rapid reversible changes in organ size as a component of adaptive behavior. Trends Ecol Evol $12: 134-138$

Ricklefs RE (1997) Comparative demography of new world populations of thrushes (Turdus spp). Ecol Monogr 67:23-43

Ricklefs RE, Bloom G (1977) Components of avian breeding productivity. Auk 94:86-96

Ricklefs RE, Wikelski M (2002) The physiology/life history nexus. Trends Ecol Evol 17:462-468

Ricklefs RE, Konarzewski M, Daan S (1996) The relationship between basal metabolic rate and daily energy expenditure in birds and mammals. Am Nat 147:1047-1071

Robinson WD, Hau M, Klasing KC, Wikelski M, Brawn JD, Austin SH, Tarwater CE, Ricklefs RE (2010) Diversification of life histories in new world birds. Auk 127:253-262

Rosenmann M, Morrison P (1974) Maximum oxygen consumption and heat loss facilitation in small homeotherms by $\mathrm{HeO} 2$. Am J Physiol 226:490-495

Schaeffer PJ, Villarin JJ, Lindstedt SL (2003) Chronic cold exposure increases skeletal muscle oxidative structure and function in Monodelphis domestica, a marsupial lacking brown adipose tissue. Physiol Biochem Zool 76:877-887

Sgueo C, Wells ME, Schaeffer PJ (2012) Acclimatization of seasonal energetics in Northern cardinals (Cardinalis cardinalis) through plasticity of metabolic rates and ceilings. J Exp Biol 215:2418-2424

Sheehan DC, Hrapchak BB (1987) HISTOTECHNOLOGY, 2nd edn. Batelle Press, Columbus

Skutch AF (1981) New studies of tropical American birds. Pub Nuttall Ornithol Club 19:1-281

Stearns SC (1992) The evolution of life histories. Oxford University Press, New York

Steiger SS, Kelley JP, Cochran WW, Wikelski M (2009) Low metabolism and inactive lifestyle of a tropical rain forest bird investigated via heart-rate telemetry. Physiol Biochem Zool 82:580-589

Stutchbury BJM, Morton ES, Piper WH (1998) Extra-pair mating system of a synchronously breeding tropical songbird. J Avian Biol 29:72-78

Swanson DL (1991) Substrate metabolism under cold stress in seasonally acclimatized dark-eyed juncos. Physiol Zool 64:1578-1592

Swanson DL, Drymalski MW, Brown JR (1996) Sliding vs. static cold exposure and the measurement of summit metabolism in birds. J Therm Biol 21:221-226

Tieleman BI, Dijkstra TH, Lasky JR, Mauck RA, Visser GH, Williams JB (2006) Physiological and behavioural correlates of life-history variation: a comparison between tropical and temperate zone House Wrens. Funct Ecol 20:491-499

Vézina F, Jalvingh KM, Dekinga A, Piersma T (2006) Acclimation to different thermal conditions in a northerly wintering shorebird is driven by body mass related changes in organ size. J Exp Biol 209:3141-3154

Vleck CM, Vleck D (1979) Metabolic rate in five tropical bird species. Condor 81:89-91

Wiersma P, Chappell MA, Williams JB (2007a) Cold- and exerciseinduced peak metabolic rates in tropical birds. Proc Nat Acad Sci USA 104:20866-20871

Wiersma P, Munoz-Garcia A, Walker A, Williams JB (2007b) Tropical birds have a slow pace of life. Proc Nat Acad Sci USA 104:9340-9345

Wikelski M, Ricklefs RE (2001) The physiology of life histories. Trends Ecol Evol 16:479-481

Williams JB, Tieleman BI (2005) Physiological adaptation in desert birds. Bioscience 55:416-425

Zheng WH, Li M, Liu JS, Shao SL (2008) Seasonal acclimatization of metabolism in Eurasian tree sparrows (Passer montanus). Comp Biochem Physiol A 151:519-525 